\title{
Approximating fuzzy preorders and equivalences. A similarity based approach.
}

\author{
Dionís Boixader and J. Recasens
}

\begin{abstract}
Although Fuzzy Preorders and Fuzzy Equivalences are intended to model vague concepts, they are defined in terms of properties or axioms to be fulfilled in a crisp way. In this paper we present two different approaches to overcome this problem (the axiomatic and the similarity based approaches) and relate them both. New results concerning the similarity between relational structures are obtained. As a consequence, every arbitrary fuzzy relation will be considered to be a fuzzy preorder or a fuzzy equivalence, at least to some extent.
\end{abstract}

\section{INTRODUCTION}

$\mathrm{F}$ UZZY preorders and equivalences are fuzzy versions of the two well known relational structures in the classical setting - preorders and equivalence relations.

Definitions for such structures were firstly proposed by Zadeh [1] and those original definitions have been generally accepted since then as the natural way to model order and equivalence in vague environments.

Fuzzy equivalences appear under a variety of names in the literature, which include terms such as similarity relations (the original as it appears in Zadeh's seminal paper), likeness relations, indistinguishability operators and fuzzy equalities.

Sometimes the differences in the names reflect the use of a particular t-norm - such as the Lukasiewicz t-norm involved in likeness relations, or the MIN t-norm used in similarities - sometimes it is simply a matter of choice for the authors [2]. We will favour the expression fuzzy equivalence relation along these pages, but at the same time similarity, proximity or some other distance related terms may occasionally appear as well, but always used in a general, t-norm free sense.

In spite of the many different names, these definitions are fully coincident in their structures. All of them are based upon the individual fuzzyfication of each of the crisp standard axioms involved - namely reflexivity, transitivity

This work was supported in part by the Spanish DGI research project TIN2009-07235

D. Boixader and J. Recasens are both with the E.T.S. d'Arquitectura del Vallès, Universitat Politècnica de Catalunya, Pere Serra 1-15, Sant Cugat del Vallès 08190 Spain. Corresp. author: dionis.boixader@upc.edu and symmetry. Whenever a fuzzy relation is reflexive and transitive in this new fuzzy sense, it is called a fuzzy preorder. If it is also symmetrical, then it is called a fuzzy equivalence or something more specific, such as likeness etc.

We will refer to that way of fuzzyfication as the axiomatic approach, because it deals exclusively with the list of axioms, neglecting some other key features of those relational structures, such as the clusters in the case of the equivalence relations.

Before presenting the reader with the central problems in this paper, let us introduce the preliminary definitions and concepts which are embodied in the axiomatic approach.

Definition 1.1: An operation $T$ on the unit interval $[0,1]$ is called a t-norm if it is associative, commutative and it satisfies the boundary conditions $T(x, 0)=T(0, x)=0$ and $T(x, 1)=T(1, x)=x$ for all $x$ in $[0,1]$.

Usually, continuity of the t-norm is also assumed. Strictly speaking, though the results presented in this paper only require that the t-norm be left continuous.

Closely related to the t-norms are their quasi-inverses, which play an important part in this paper.

Definition 1.2: Given a t-norm $T$, its quasi-inverse $I_{T}$ or $\vec{T}$ is the following operation on the unit interval:

$$
I_{T}(x, y)=\vec{T}(x \mid y)=\sup \{a \in[0,1] / T(a, x) \leq y\}
$$

for all $x$ and $y$ in $[0,1]$.

By making the quasi-inverse symmetrical we obtain another operation on the unit interval, the natural equivalence.

Definition 1.3: Given a t-norm $T$, its associated natural equivalence $E_{T}$ or $\vec{T}$ on the unit interval is:

$$
E_{T}(x, y)=\vec{T}(x, y)=\min \{\vec{T}(x \mid y), \vec{T}(y \mid x)\} \text { for every } x
$$
and $y$ in $[0,1]$.

The operations we have just introduced are generally viewed as the conjunction (the t-norm), the residuated implication (the quasi-inverse) and the biimplication or equivalence (the natural equivalence) in the setting of Fuzzy 
Logic.

Definition 1.4: A fuzzy relation $R$ on a universe $X$, i.e. a mapping $R: X \times X \rightarrow[0,1]$, is said to be:

Reflexive, if $R(x, x)=1$ for every $x$ in $X$.

Symmetrical, if $R(x, y)=R(y, x)$ for every $x$ and $y$ in $X$.

Transitive with respect to a given t-norm $T$, or $T$ transitive, if $T(R(x, y), R(y, z)) \leq R(x, z)$ for every $x, y$ and $z$ in $X$.

Definition 1.5: A fuzzy preorder is a reflexive and $T$ transitive fuzzy relation.

Definition 1.6: A fuzzy equivalence is a reflexive, symmetrical and $T$-transitive fuzzy relation.

There are mainly two different ways to grasp the meaning behind definition 1.6. First, by comparing the fuzzy equivalences to the crisp ones, which naturally brings notions such as cluster, classification and prototype, and raises questions about the many possible ways to extend those concepts to the fuzzy domain. And second, by linking them to a metric structure. This is achieved by transforming T-transitivity into the triangle inequality for distances, that is $d(x, z) \leq d(x, y)+d(y, z)$, in which the standard sum of real numbers play a role similar to that of the t-norm $T$. The order is then reversed, so that equivalence degrees near 1 correspond to distances near 0 . Thus, points linked by high degrees of equivalence become close points.

It is clear that any given fuzzy relation $S$ might meet, or fail to meet, a particular requirement among those stated in Definition 1.4 only in a precise, crisp sort of way.

Thus, a fuzzy relation $S$ such that $S(x, x)=0.9$ for some element $x$ will no longer be reflexive. Or $S$ will fail to be transitive if $S(x, y)=S(y, z)=1$ but $S(x, z)=0.9$, no matter which t-norm we try.

Furthermore, it is not possible to compare two fuzzy relations $R$ and $S$ to conclude that, for example, $R$ behaves more like a fuzzy preorder than $S$, or that $S$ is slightly more symmetric than $R$. Being a fuzzy preorder, or being symmetric for that case, is not a matter of degree. As a consequence, fuzziness itself remains excluded from all those definitions, a feature hardly desirable in any fuzzy theory.

Such claims were first made in Gottwald [3] and, since then, similar objections have been raised in [4],[5], [7] and more recently in [6]. The latter contribution by Behounek et $a l$. is part of an ambitious project which aims at introducing degrees in every single fuzzy theory as a result of a formal logic based set theory. Although the work which we present here has been independently developed, we must acknowledge that many results can be found in [6], although they are there presented under the language of formal logic, while our work has been mostly inspired by the metric notion of proximity and it is presented accordingly.

In our approach, we propose two different lines of action. The fist one consists in relaxing the definitions of reflexivity, transitivity and symmetry by introducing degrees. This is part of the axiomatic approach, and it is found under slightly different forms in all the previous references.

The second line of action deals with the notion of similarity between relational structures, and makes no reference at all to the list of axioms. It is this second view which is essentially built on metric ideas.

Many measures to compare fuzzy sets, or fuzzy relations, may be found in the literature [8]. We will remain focused on pointwise similarity. That means, every pair of numeric values $R(x, y)$ and $S(x, y)$ are compared to infer that, as a whole, $R$ and $S$ are at least so dissimilar as those two. We will refer to that particular measure of similarity as the natural equivalence too, because it extends Definition 1.3 in a natural way. The term natural indistinguishability is also used in $[2,4]$.

Definition 1.7: Given two fuzzy relations $R$ and $S$ on $X$, the natural equivalence or indistinguishability between them is $\mathcal{E}_{T}(R, S)=\inf _{x, y \in X} E_{T}(R(x, y), S(x, y))$

When presented with an arbitrary relation $S$, we may try to find proper fuzzy preorders or equivalences $R$ which are close enough to $S$ when compared through $\mathcal{E}_{T}(R, S)$. The degree of similarity between $S$ and $R$ may be then regarded as the extent to which $S$ is a fuzzy preorder or equivalence. For obvious reasons, we will refer to $S$ as the candidate relation, while $R$ will be called a reference relation.

Sometimes, the two relations' specifity will be taken into consideration as well as their closeness. We will then compare the two through the so-called natural or pointwise order.

Definition 1.8: Two fuzzy relations $R$ and $S$ on $X$ are said to be pointwise ordered, $R \leq_{X} S$, if $R(x, y) \leq S(x, y)$ for all $x, y$ in $X$.

The outcomes of the two approaches - the axiom based and the similarity based - are pretty much the same. Every single fuzzy relation $S$ is assigned a degree of membership to the target class - the fuzzy preorders or the fuzzy equivalences. Therefore, these categories become fuzzy sets of the set of all fuzzy relations, rather than crisp ones. 
However, the two approaches are not exactly equivalent, and the problem arises of relating them in an effective way. On the one hand, once a reference relation $R$ has been proposed for a given candidate relation $S$, lower bounds for reflexivity, transitivity and symmetry ought to be computed for $S$ based on the degree of similarity $\mathcal{E}_{T}(R, S)$. On the other hand, if $S$ is known to meet the axioms only to some extent, then the problem is about finding a reference relation $R$ in the neighbourhood, their closeness $\mathcal{E}_{T}(R, S)$ being determined by the degrees of reflexivity, transitivity and symmetry of $S$.

The contributions in this paper are related to the second half of the problem - namely, finding reference relations $R$ in the neighbourhood of the candidate $S$. Section 2 contains the preliminaries and some known results. Section 3 analyses the role of the transitive closure as a reference relation, and Section 4 proposes reference relations based on the representation theorems.

\section{SOME KNOWN RESULTS}

Let us recall some definitions which are related to the grading of the standard axioms.

Definition 2.1: A fuzzy relation $R$ on a universe $X$, is said to be:

$a$-Reflexive, if $R(x, x) \geq a$ for a given $a$ in [0,1], and for every $x$ in $X$.

a-Symmetric with respect to a given t-norm $T$ if $T(a, R(x, y)) \leq R(y, x)$ for a given $a$ in $[0,1]$, and for every $x$ and $y$ in $X$.

$a$-Transitive with respect to a given t-norm $T$, or $a-T$ transitive, if $T(a, T(R(x, y), R(y, z))) \leq R(x, z)$ for a given $a$ in $[0,1]$, and for every $x, y$ and $z$ in $X$.

Two easy consequences follow from Definition 2.1. First, when $a=1$ the standard reflexivity, symmetry and $T$ transitivity for fuzzy relations are retrieved. Second, every $a$-reflexive, $a$-symmetric or $a$ - $T$-transitive fuzzy relation is also $b$-reflexive, $b$-symmetric, $b$-transitive for any $b \leq a$.

As a consequence, degrees for these properties can be defined in a natural way for any given fuzzy relation.

Definition 2.2: For any t-norm $T$ and any fuzzy relation $R$ on a universe $X$ :

Its degree of reflexivity is $a_{r}=\inf _{x \in X} R(x, x)$

Its degree of symmetry w.r.t. $T$ is

$$
a_{s}=\inf _{x, y \in X}\{\sup \{a \in[0,1] / T(a, R(x, y)) \leq R(y, x)\}\}
$$

Its degree of transitivity w.r.t. $T$ is

$$
a_{t}=\inf _{x, y \in X}\{\sup \{a \in[0,1] / T(a, T(R(x, y), R(y, z)) \leq R(x, z)\}\}
$$

A fuzzy relation $R$ is a fuzzy preorder if, and only if, $a_{r}=a_{t}=1$, and it is a fuzzy equivalence if, and only if, $a_{r}=a_{s}=a_{t}=1$

The previous degrees can be written in terms of $I_{T}$, the quasi-inverse or residuated implication associated to the tnorm $T$. By using this implication,

- the degree of symmetry of $R$ w.r.t. $T$ is

$a_{s}=\inf _{x, y \in X}\left\{I_{T}(R(x, y), R(y, x)\}\right.$, and

- the degree of transitivity of $R$ w.r.t. $T$ is

$a_{t}=\inf _{x, y \in X}\left\{I_{T}(T(R(x, y), R(y, z)), R(x, z))\right\}$

A possible alternative is to rewrite $a_{s}$ in terms of $E_{T}$ as follows: $a_{s}=\inf _{x, y \in X} E_{T}(R(x, y), R(y, x))$

Definition 2.3: A fuzzy relation is said to be an $a$-Fuzzy Preorder if its levels of reflexivity and transitivity are both above the threshold $a$.

Definition 2.4: A fuzzy relation is said to be an $a-F u z z y$ Equivalence if its levels of reflexivity, symmetry and transitivity are all above the threshold $a$.

From now on, we will assume the reference relation $R$ is either a fuzzy preorder or equivalence, while for $S$, the candidate relation, we do not ask any particular requirement but that of being close enough to $R$ when the two are compared by $E_{T}$. We are going to study how reflexive, symmetric or $T$-transitive $S$ is, owing to its proximity to the reference relation $R$.

Proposition 2.5: Let $R$ and $S$ be fuzzy relations on $X$. If $R$ is reflexive and $E_{T}(R, S) \geq a$ then $S$ is $a$-reflexive.

Proposition 2.6: Let $R$ and $S$ be fuzzy relations on $X$. If $R$ is symmetrical and $E_{T}(R, S) \geq a$ then $\mathrm{S}$ is $b$-symmetrical, with $b=T(a, a)$.

Proposition 2.7: Let $\mathrm{R}$ and $\mathrm{S}$ be fuzzy relations on $X$. If $R$ is $T$-transitive and $E_{T}(R, S) \geq a$ then $S$ is $b$-T-transitive, with $b=T(T(a, a), a)$.

Summarizing, when $R$ is a fuzzy preorder or equivalence, we have: 
Proposition 2.8: If $R$ is a fuzzy preorder (resp. equivalence) on $X$, and $S$ is a fuzzy relation such that $E_{T}(R, S) \geq a$, then $S$ is a $b$-fuzzy preorder (resp. $b$ equivalence) with $b=T(T(a, a), a)$.

\section{TRANSITIVE CLOSURE AS A REFERENCE RELATION}

The transitive closure $\bar{S}$ of an arbitrary fuzzy relation $S$ is the lowest $T$-transitive relation which is above $S$ with respect to the pointwise order $\left(S \leq_{X} \bar{S}\right)$ ([9] for example).

When looking for a reference relation in the neighbourhood of $S$ the transitive closure seems to be a choice natural enough. However, being the closest among the set of all the $T$-transitive relations which are above $S$ does not mean that it is going to be very close.

In [4] it is pointed out that the natural equivalence $\mathcal{E}_{T}$ between $S$ and $\bar{S}$ may fall, generally speaking, below the degree of transitivity of $S$. There, the similarity between $S$ and $\bar{S}$ is referred to as the strong transitivity degree of $S$.

Definition 3.1: The strong transitivity degree $A_{t}$ of a fuzzy relation $S$ with respect to a t-norm $T$ is $A_{t}=\mathcal{E}_{T}(\bar{S}, S)$

Proposition 4.2 [4]: If $a_{t}$ and $A_{t}$ are respectively the transitivity degree and the strong transitivity degree of an arbitrary relation $S$ with respect to a t-norm $T$, then $A_{t} \leq a_{t}$.

First thing we are going to show is that it is perfectly possible to have a situation where $A_{t}<a_{t}$.

Example 4.3: $\quad T_{L}(a, b)=\max \{a+b-1,0\}$ stands for the Lukasiewicz t-norm. We will follow the exponential like notation $a^{2}=T_{L}(a, a), \quad a^{3}=T_{L}\left(T_{L}(a, a), a\right)$ and so forth, instead of writing many nested $T_{L}$ symbols.

Let us consider a reflexive and symmetrical fuzzy relation $S$ on the finite set $X=\left\{x_{1}, x_{2}, x_{3}, x_{4}\right\}$ defined as follows:

$$
\begin{aligned}
& S\left(x_{i}, x_{i+1}\right)=d \\
& S\left(x_{i}, x_{i+2}\right)=T_{L}\left(d^{2}, a\right) \\
& S\left(x_{i}, x_{i+3}\right)=T_{L}\left(d^{3}, a^{2}\right)
\end{aligned}
$$

where $i, \mathrm{i}+1, i+2, i+3$ are integers between 1 and 4 , and the real numbers $a, d \in(0,1)$ are chosen in a way that $S>0$.

For instance, $a=0.9$ and $d=0.9$ will result in:

$$
\begin{aligned}
& S\left(x_{1}, x_{2}\right)=S\left(x_{2}, x_{3}\right)=S\left(x_{3}, x_{4}\right)=0.9 \\
& S\left(x_{1}, x_{3}\right)=S\left(x_{2}, x_{4}\right)=T_{L}(0.8,0.9)=0.7 \\
& S\left(x_{1}, x_{4}\right)=T_{L}(0.7,0.8)=0.5
\end{aligned}
$$

Here $d$ stands for the similarity between two consecutive elements, and it is easy to check that $a$ is the transitivity degree with respect to $T_{L}$. If we then compute the transitive closure we will find that $\bar{S}\left(x_{1}, x_{4}\right)=0.7$ which differs from the original $S$ in $E_{L}(0.5,0.7)=0.8$ That is the maximum difference of all, so that $A_{t}=\mathcal{E}_{L}(\bar{S}, S)=0.8<0.9=a_{t}$.

The general rule behind this numerical example is that $S\left(x_{1}, x_{4}\right)=T_{L}\left(d^{3}, a^{2}\right) \quad$ while $\quad \bar{S}\left(x_{1}, x_{4}\right)=d^{3}, \quad$ and $A_{t}=E_{L}(\bar{S}, S)=a^{2}$ follows, provided that $0<T_{L}\left(d^{3}, a^{2}\right)<1$

Further generalization is obtained if we work out the same kind of example on a set $X=\left\{x_{1}, x_{2}, \ldots, x_{n}\right\}$. Then we will obtain $S\left(x_{0}, x_{n}\right)=T_{L}\left(d^{n-1}, a^{n-2}\right)$ while $\bar{S}\left(x_{0}, x_{3}\right)=d^{3}$. This proves that the strong degree of transitivity $A_{t}$ may in some cases reach the value $A_{t}=\mathcal{F}_{L}(\bar{S}, S)=a^{n-2}$ which is well below the standard degree of transitivity which is $a_{t}=a$.

Next is the central result in this section. It states that the transitive closure cannot lie any farther away than it does in the previous example.

Theorem 4.4: Let $S$ be an $a$-T-transitive relation on a finite set $X=\left\{x_{1}, x_{2}, \ldots, x_{n}\right\}$, and $A_{t}=E_{L}(\bar{S}, S)$ its strong transitivity degree. Then $A_{t} \leq a^{n-2}$.

We will present the reader with the outline of a proof for theorem 4.4 which makes extensive use of chains. A chain of length $k$ starting on $x_{\mathrm{i}}$ and ending on $x_{\mathrm{j}}$ is an ordered set $x_{i}=u_{1}, u_{2}, \ldots u_{k}=x_{j}$ with all the $u_{\mathrm{m}}$ belonging to $X$.

Given a chain $C$ of length $k \geq 4$ connecting $x_{\mathrm{i}}$ with $x_{\mathrm{j}}$ it may happen that the transitivity along the chain fall below the transitivity degree $a_{t}$, so that:

$S\left(x_{i}, x_{j}\right)=T\left(\varepsilon_{C}, T\left(S\left(x_{i}, u_{2}\right) \ldots T\left(S\left(x_{k-2}, u_{k-1}\right), S\left(u_{k-1}, x_{j}\right)\right) \ldots\right)\right)$ with $\varepsilon_{C}<a_{t}$ (note that this was just what happened in example 4.3).

Lemma 4.5: $\varepsilon_{C} \geq a_{t}^{k-2}$

Every chain linking the two elements will have its own $\varepsilon_{C}$ and then we can consider the lowest of all these values:

$$
\varepsilon\left(x_{i}, x_{j}\right)=\inf \left\{\varepsilon_{C} / C \in C\left(x_{i}, x_{j}\right)\right\} \text { where } C(x, y) \text { stands }
$$

for the set of all chains of any length starting on $x_{i}$ and ending on $x_{j}$.

Now, we can modify $S$ by increasing it with the values $\varepsilon\left(x_{i}, x_{j}\right)$ so that transitivity be restored in every possible 
chain, that is: $\tilde{S}(x, y)=I_{T}\left(\varepsilon\left(x_{i}, x_{j}\right), S\left(x_{i}, x_{j}\right)\right)$

Lemma 4.6: $\mathcal{E}_{L}(\tilde{S}, S) \geq a_{t}^{n-2}$

(Lemma 4.6 is an easy consequence of lemma 4.5)

Lemma 4.7: $\tilde{S}$ is $T$-transitive.

(Lemma 4.7 is not difficult to prove)

Finally, as a consequence of $\tilde{S}$ being $T$ - transitive we have that $\tilde{S} \geq \bar{S} \geq S$, and lemma 4.5 completes a proof for theorem 4.4 .

Although it is not essential for the ideas in this section, the following proposition has some theoretical interest because it provides a way of computing transitive closures which, to the best of our knowledge, is new.

\section{Proposition $4.8 \tilde{S}=\bar{S}$}

\section{REFERENCE RELATIONS OBTAINED VIA REPRESENTATION THEOREMS}

Representation theorems play a crucial role in the field of Fuzzy Relations. Valverde's Representation Theorems for Fuzzy Preorders and Equivalences [10] are the best known ones, although it is worth mentioning an earlier version of such theorems by Ovchinnikov [11], as well as a later generalization by Fodor and Roubens [12].

Representation theorems provide ways for obtaining a given fuzzy relation starting from a family of fuzzy sets. This process is often referred to as generating the relation, and the involved fuzzy sets are called accordingly generators.

Theorem 5.1 (Valverde's Representation Theorem for fuzzy preorders): A fuzzy relation $R$ on a set $X$ is a fuzzy preorder if, and only if, there exists a family $\left\{h_{i}: X \rightarrow[0,1]\right\}_{i \in I}$ of fuzzy subsets of $X$ such that $R(x, y)=\inf _{i \in I} I_{T}\left(h_{i}(x), h_{i}(y)\right)$

Theorem 5.2 (Valverde's Representation Theorem for fuzzy equivalences): A fuzzy relation $R$ on a set $X$ is a fuzzy equivalence if, and only if, there exists a family $\left\{h_{i}: X \rightarrow[0,1]\right\}_{i \in I}$ of fuzzy subsets of $X$ such that $R(x, y)=\inf _{i \in I} E_{T}\left(h_{i}(x), h_{i}(y)\right)$

Although these theorems deal only with the existence of the generating families $\left\{h_{i}\right\}_{i \in I}$ and they do not provide any way for obtaining such families of fuzzy subsets, their proofs do. Such proofs are built upon the fact that the columns of
$R$, that is the family of fuzzy sets $\left\{h_{x}\right\}_{x \in X}$ where each $h_{x}$ is defined by $h_{x}(y)=R(x, y)$, are a generating family.

Using the whole set of columns, which is nothing less than the whole relation $R$, in order to obtain $R$ itself does not look like any impressive achievement. However, it is important to realize that theorems 5.1 and 5.2 apply only to those relations $R$ that already are fuzzy preorders or fuzzy equivalences, that is, they apply only to reference relations. If we start with a candidate relation $S$ which is not a proper fuzzy preorder or equivalence, what we obtain by using the set of its columns as generators is not $S$ itself, but an entirely different fuzzy relation $R$, which is a proper fuzzy preorder or equivalence, according to the same theorems. The question we may ask now is: how different is $R$ from the original $S$ ?

Proposition 5.3 Let $S$ be an $a$-reflexive, $b$-transitive relation on $X$, and let $R$ be the relation defined by:

$$
R(u, v)=\inf _{x \in X} I_{T}(S(x, u), S(x, v))
$$

for each pair $u, v$ in $X$. Then:

5.3.1 $R$ is a fuzzy preorder.

5.3.2 $\mathcal{E}_{T}(R, S) \geq T(a, b)$

5.3.1 follows as an immediate consequence of theorem 5.1 , when the generating family used is $\left\{h_{x}\right\}_{x \in X}$ with $h_{x}(y)=R(x, y)$.

5.3.2 is not an straightforward result. It is obtained through a series of steps which may be plainly stated but require of somehow technical proofs nonetheless.

An outline of the proof is as follows.

First, we prove that $T(b, S(u, v)) \leq S(u, v) \leq I_{T}(a, S(u, v))$

Second, the same inequalities are shown to be true for $I_{T}(S(x, u), S(x, v))$ for each $x$ in $X$, and therefore:

$$
\begin{aligned}
& T(b, S(u, v)) \leq \inf _{x \in X} I_{T}(S(x, u), S(x, v))= \\
& R(x, y) \leq I_{T}(a, S(u, v))
\end{aligned}
$$

Finally, since we have managed to put both $S$ and $R$ between the same upper and lower bounds, it follows that:

$E_{T}(S(u, v), R(u, v)) \geq E_{T}\left(T(b, S(u, v)), I_{T}(a, S(u, v))\right)=T(a, b)$ which proves that $\mathcal{E}_{T}(R, S) \geq T(a, b)$.

It is important to notice that the relation $R$ obtained in the present way is neither less nor more specific than $S$ when the pointwise order $\leq_{X}$ is taken into consideration. 


\section{CONCLUSION}

Every single fuzzy relation $S$ may be considered to be a fuzzy preorder or a fuzzy equivalence to some extent, or approximately.

For an arbitrary relation $S$ the degree it may be assigned as a member of the class of all fuzzy preorders or equivalences is closely related to the existence of a proper fuzzy preorder or equivalence $R$ in the neighbourhood. The closer $R$ is to $S$, the higher de degree.

In this paper we have proposed two possible choices for $R$, which are the transitive closure, and a relation obtained via the representation theorems.

While the transitive closure has the advantage of being less specific than the proposed relation $S$, it has been shown that it may lie quite away from it. On the other hand, the kind of relations obtained via the representation theorems are closer to the original $S$, but they fail to show any regular behaviour regarding specificity.

\section{REFERENCES}

[1] L. A. Zadeh, "Similarity relations and fuzzy orderings," Information Sciences, 3, 1971, pp. 177-200.

[2] D. Boixader, J. Jacas and J. Recasens, "Fuzzy equivalence relations: advanced material" in Fundamentals of Fuzzy Sets, D. Dubois and H. Prade eds, Kluwer 2000, pp.261-290.

[3] S. Gottwald, "Fuzzified fuzzy relations. Proc. of the 4th IFSA congress, vol. Mathematics, Brussels 1991, pp.82-86.

[4] J. Jacas and J. Recasens, "Fuzzified properties of fuzzy relations" Proc. IPMU 10th International Conference, 2002, pp.157-161.

[5] L. Behounek, "Extensionality in graded properties of fuzzy relations" Proc. IPMU Eleventh 10th International Conference, vol.II, 2006, pp.1604-1611.

[6] L. Behounek, Bodenhofer and Cintula "Relations in Fuzzy Class Theory - initial steps" FSS 159 (2008) pp.1729-1772.

[7] D. Boixader and J. Recasens, "Approximate Fuzzy Preorders and Equivalences" Proc. FuzzIEEE 2009, Korea.

[8] B. Bouchon-Meunier, M. Rifqi, S. Bothorel "Towards General Measures of Comparison of Objects" Fuzzy Sets and Systems, 84, Issue 2, 1996, pp. 143-153.

[9] G. J. Klir and Bo Yuan, Fuzzy sets and fuzzy logic. Theory and applications. New Jersey: Prentice Hall, 1995, ch. 3.

[10] L. Valverde, "On the Structure of F-indistinguishability Operators ", Fuzzy Sets and Systems, 17, 1985, pp.313-328.

[11] S.V. Ovchinnikov, "Structure of fuzzy binary relations" Fuzzy Sets and Systems , 6, 1981, pp. 169-185.

[12] J.C. Fodor, M. Roubens "Structure of Transitive Valued Binary Relations" Mathematical Social Sciences, 30, 1995, pp.71-94. 